\title{
AVALIAÇÃO DE SEMEADORA-ADUBADORA DE PRECISÃO TRABALHANDO EM TRÊS SISTEMAS DE PREPARO DO SOLO
}

\author{
CARLOS E. A. FURLANI', AFONSO LOPES ${ }^{1}$, ROUVERSON P. DA SILVA ${ }^{1}$
}

RESUMO: Avaliou-se uma semeadora-adubadora de precisão com quatro linhas, na cultura do milho (Zea mays L.), trabalhando em Latossolo Vermelho-Escuro eutrófico, A moderado, textura argilosa. As parcelas experimentais foram dispostas em blocos casualizados, com quatro repetições, em esquema fatorial $3 \times 3$, totalizando 36 observações. Os fatores foram dados pelos sistemas de preparo do solo (convencional, plantio direto e reduzido) e pelas marchas do trator ( $3^{\mathrm{a}}$ reduzida alta, $4^{\mathrm{a}}$ reduzida baixa e $4^{\mathrm{a}}$ reduzida alta). Foram analisadas as variáveis velocidade de deslocamento, queda de velocidade, força de tração e potência na barra, consumo de combustível, rotação do motor e patinagem média dos rodados do trator. Os resultados mostraram que a operação de semeadura em solo sob preparo reduzido apresentou maior consumo de combustível, menor velocidade de deslocamento, menor capacidade de campo efetiva e maior patinagem.

PALAVRAS-CHAVE: desempenho, velocidade, combustível.

\section{EVALUATION OF THE PERFORMANCE OF A PRECISION SEEDER WORKING IN THREE TILLAGE SYSTEMS}

\begin{abstract}
The performance of a precision seeder with four lines in the culture of maize (Zea mays L.) working in Latossolo Vermelho Escuro Eutrófico, moderate A, argillaceous texture was evaluated. The experimental parcels were disposed in casualized blocks with four repetitions, according to a factorial scheme $3 \times 3$, totalizing 36 observations. The factors had been given by the systems of tillage (conventional, no tillage and reduced) and by the gears of the tractor $\left(3^{\text {rd }}\right.$ reduced high, $4^{\text {th }}$ reduced low and $4^{\text {th }}$ reduced high). The variables analyzed were: displacement speed, speed reduction, traction force and drawbar pull, fuel consumption, motor rotation and medium slipping wheels of the tractor. The results showed that the sowing operation in soil under reduced tillage presented larger fuel consumption, smaller displacement speed, smaller effective field capacity and larger slipping.
\end{abstract}

KEYWORDS: performance, speed, fuel.

\section{INTRODUÇÃO}

O preparo do solo tem como finalidade proporcionar condições favoráveis para o desenvolvimento adequado das culturas, porém a qualidade dos mesmos, além de influenciar nessas culturas, também pode auxiliar ou prejudicar o desempenho das semeadoras-adubadoras. Assim, tornase fundamental avaliar como essas máquinas trabalham em diferentes condições de solo.

Segundo a norma EP 291.1 (ASAE, 1997), existem diversos sistemas de preparo do solo, os quais são enquadrados em categorias definidas como: a) convencional, que é a combinação de duas ou mais operações, b) reduzido, uma única operação e c) plantio direto, ou seja, semeadura em solo nãopreparado.

\footnotetext{
${ }^{1}$ Prof. Dr., Departamento de Engenharia Rural, Faculdade de Ciências Agrárias e Veterinárias, UNESP, Câmpus de Jaboticabal, Via de Acesso Prof. Paulo Donato Castellane, km 5, furlani@fcav.unesp.br

Recebido pelo Conselho Editorial em: 5-3-2004

Aprovado pelo Conselho Editorial em: 21-2-2005
} 
Semeadura é a operação de implantação de culturas que utilizam sementes como órgãos de propagação. A máquina que realiza a semeadura de culturas cujas sementes são graúdas (milho, soja, feijão, entre outras), dosando-as espaçadas, de acordo com a recomendação agronômica, é denominada de semeadora de precisão (GADANHA JÚNIOR et al., 1991).

SIQUEIRA et al. (2001), trabalhando com semeadora-adubadora (plantio direto) de seis linhas e haste parabólica, obtiveram 13,14 kN de exigência de força de tração na barra. MARQUES (1999) encontrou valores de $8,47 \mathrm{kN}$ de força de tração na barra para semeadora de quatro linhas, com mecanismo de deposição de adubo tipo disco duplo concêntrico.

A força de tração na barra requerida por linha de semeadura está na faixa de 1,1 a 2,0 $\mathrm{kN}$ (ASAE, 1996). SILVA (2000) constatou que, na semeadura direta de milho (quatro linhas), os maiores valores de força de tração $(16,12 \mathrm{kN})$ ocorreram na maior velocidade. Na média do ensaio, o autor encontrou 1,65 kN (semeadura da soja) e 2,92 kN (semeadura de milho) de exigência de força de tração na barra por linha.

LEVIEN et al. (1999) constataram maior patinagem das rodas motrizes do trator na semeadura em solo arado e escarificado, comparado ao sistema plantio direto. Os mesmos autores obtiveram média de 2,1 ha $\mathrm{h}^{-1}$ de capacidade de campo e 5,90 $\mathrm{L} \mathrm{ha}^{-1}$ de consumo de combustível por área trabalhada, enquanto MARQUES et al. (1999) encontraram 1,45 ha h${ }^{-1}$ e 7,30 L ha ${ }^{-1}$, respectivamente.

FURLANI (2000), utilizando semeadora-adubadora com seis linhas (feijão), encontrou valores de 14,8; 9,4 e 6,2\% de patinagem das rodas do trator, 9,3; 7,6 e 7,1 $\mathrm{L} \mathrm{ha}^{-1}$ de consumo por área e 13,8; 11,3 e 12,0 kW de potência para solo escarificado, arado/gradeado e plantio direto, respectivamente.

MARQUES (2002) encontrou valores médios de 19,53; 22,97 e 20,30 kN de exigência de força de tração na operação de semeadura de soja (seis linhas), em preparo convencional, plantio direto e preparo reduzido, respectivamente.

O objetivo do presente trabalho foi avaliar o desempenho de semeadora-adubadora de precisão com 4 linhas, na semeadura do milho, em função do preparo do solo e de três marchas do trator.

\section{MATERIAL E MÉTODOS}

O experimento foi conduzido na Fazenda de Ensino, Pesquisa e Produção da Faculdade de Ciências Agrárias e Veterinárias, UNESP, Jaboticabal - SP, em área do Departamento de Engenharia Rural, localizado nas coordenadas geográficas $21^{\circ} 14^{\prime} 48^{\prime \prime}$ latitude sul e $48^{\circ} 16^{\prime} 44$ " longitude oeste, com altitude média de $559 \mathrm{~m}$. O solo é classificado como Latossolo Vermelho-Escuro eutrófico, A moderado, textura argilosa e relevo suave ondulado (EMBRAPA, 1999).

Os sistemas de preparo foram: a) convencional: combinação de uma aração e duas gradagens niveladoras; b) plantio direto: semeadura em solo não-preparado, e c) preparo reduzido: escarificador combinado com disco de corte e rolo destorroador. As marchas utilizadas foram definidas em função do conjunto trator-semeadora: a) terceira reduzida alta (M1); b) quarta reduzida baixa (M2), e c) quarta reduzida alta (M3), cujas velocidades teóricas, conforme o fabricante, são: 7,3; 8,1 e 10,5 $\mathrm{km} \mathrm{h}^{-1}$, respectivamente. No início de cada parcela, as rotações do motor do trator atingiram o valor máximo (próximo a $2.350 \mathrm{rpm}$ ).

Os equipamentos utilizados no preparo do solo foram: arado reversível montado, com três discos de 660 mm (26") de diâmetro e largura de corte de 0,9 m, marca Marchesan; grade niveladora de arrasto com 32 discos lisos de 458 mm (18") de diâmetro e largura de trabalho de 2,77 m, marca Piccin, e escarificador de sete hastes parabólicas, conjugadas com rolo destorroador e discos de corte, com espaçamento de $30 \mathrm{~cm}$ entre hastes e largura de trabalho de 2,10 m, marca Marchesan. 
A semeadora-adubadora de precisão utilizada foi da marca Marchesan, modelo Cop Suprema, equipada com quatro linhas espaçadas de $0,90 \mathrm{~m}$, capacidade de adubo de $1.310 \mathrm{~kg}$ e de semente de $200 \mathrm{~kg}$ (durante o experimento, mantiveram-se os depósitos com aproximadamente meia carga), largura útil de 3,6 m e sulcador de adubo tipo facão, sendo a profundidade média de trabalho de $8 \mathrm{~cm}$.

O trator utilizado para tracionar os equipamentos de preparo foi da marca Massey Fergusson, modelo MF-620, 4x2 TDA com 72,3 kW (105 cv) de potência no motor. Para tracionar a semeadoraadubadora, utilizou-se de trator instrumentado da marca Valtra, modelo BM-100, 4x2 TDA com $73,6 \mathrm{~kW}(100 \mathrm{cv})$ de potência no motor. A distribuição de peso do trator instrumentado foi de $40 \mathrm{e}$ $60 \%$, respectivamente, para os eixos dianteiro e traseiro, com lastragem máxima e massa total em ordem de marcha de $5.400 \mathrm{~kg}$.

A determinação da força de tração na barra foi obtida por meio de célula de carga M. Shimizu, modelo TF 400, colocada entre a barra de tração do trator e o cabeçalho da semeadora-adubadora. A rotação do motor do trator foi obtida de forma indireta, ou seja, determinou-se a rotação da tomada de potência por meio de sensor de rotação marca S\&E Instrumentos de Testes e Medições Ltda., modelo GIDP-60-U-12v, e com a relação de transmissão motor/TDP de 3,47.

Determinou-se a diminuição da velocidade real de deslocamento em relação à teórica fornecida pelo fabricante, visando a avaliar o aproveitamento da velocidade nominal, em função dos sistemas de preparo do solo e das marchas selecionadas. Calculou-se esse parâmetro pela eq.(1).

$$
A v_{\text {nom }}=100\left(1-\frac{\mathrm{V}_{\mathrm{r}}}{\mathrm{V}_{\mathrm{t}}}\right)
$$

em que,

$A v_{n o m}$ - aproveitamento da velocidade nominal, \%;

$\mathrm{V}_{\mathrm{r}}$ - velocidade real de deslocamento, $\mathrm{km} \mathrm{h}^{-1}$, e

$\mathrm{V}_{\mathrm{t}}$ - velocidade teórica ou nominal, $\mathrm{km} \mathrm{h}^{-1}$.

A capacidade de campo efetiva foi determinada em função da largura útil de trabalho da semeadora e da velocidade real de deslocamento, usando-se a eq.(2).

$$
\text { Ccef }=\frac{\mathrm{V}_{\mathrm{r}} \mathrm{L}_{\mathrm{t}}}{10}
$$

em que,

Ccef - capacidade de campo efetiva, ha $\mathrm{h}^{-1}$, e

$\mathrm{L}_{\mathrm{t}}$ - largura de trabalho útil da semeadora, $\mathrm{m}$.

Determinou-se a potência na barra de tração pela eq.(3).

$$
\mathrm{P}_{\mathrm{bt}}=\mathrm{F}_{\mathrm{bt}} \mathrm{V}_{\mathrm{r}}
$$

em que,

$\mathrm{P}_{\mathrm{bt}}$ - potência na barra de tração, $\mathrm{kW}$;

$\mathrm{F}_{\mathrm{bt}}$ - força de tração demandada pela semeadora, $\mathrm{N}$, e

$\mathrm{V}_{\mathrm{r}}$ - velocidade real de deslocamento, $\mathrm{m} \mathrm{s}^{-1}$.

O consumo horário de combustível foi obtido com auxílio de equipamento desenvolvido por LOPES et al. (2003). Para determinar o consumo por área, utilizou-se da equação 4.

$$
\mathrm{C}_{\text {area }}=\frac{\mathrm{C}_{\text {hor }}}{\text { Ccef }}
$$


em que,

Cárea - consumo de combustível por área, $\mathrm{L} \mathrm{ha}^{-1}$, e

$\mathrm{C}_{\text {hor }}$ - consumo de combustível horário, $\mathrm{L} \mathrm{h}^{-1}$.

O consumo específico de combustível foi calculado com base na eq.(5).

$$
\mathrm{C}_{\text {esp }}=\frac{\mathrm{d} \mathrm{C}_{\text {hor }}}{\mathrm{P}_{\mathrm{bt}}}
$$

em que,

Cesp - consumo específico de combustível, $\mathrm{g} \mathrm{kWh}^{-1}$, e

d - densidade do combustível, $\mathrm{g} \mathrm{L}^{-1}$.

Para estimar a patinagem dos rodados do trator, utilizou-se de sensores modelo GIDP-60-12v em cada uma das rodas e obteve-se a média dos giros para o trator, sem e com carga. Logo, calculou-se a porcentagem da patinagem pela eq.(6).

$$
\mathrm{P}_{\mathrm{i}}=\frac{(\mathrm{nc}-\mathrm{ns}) 100}{\mathrm{nc}}
$$

em que,

$\mathrm{P}_{\mathrm{i}}$ - porcentagem da patinagem, $\%$;

nc - número médio dos giros das rodas com carga, e

ns - número médio dos giros das rodas sem carga.

O delineamento estatístico adotado foi o de blocos casualizados, em esquema fatorial 3 x 3 (três sistemas de preparo do solo e três marchas do trator), com quatro repetições. Os dados obtidos foram submetidos à análise de variância, e as médias foram comparadas de acordo com o teste de Tukey, para $5 \%$ de probabilidade.

\section{RESULTADOS E DISCUSSÃO}

Pela Tabela 1, pode-se verificar que a velocidade de deslocamento do conjunto trator-semeadora apresentou diferença estatística para as marchas utilizadas, o que era o intuito dessa escolha, porém observa-se que, na semeadura em solo com preparo reduzido, a velocidade foi menor, cerca de $6 \%$. As três marchas estudadas forneciam velocidades teóricas de 7,3 (M1); 8,1 (M2) e 10,5 $\mathrm{km} \mathrm{h}^{-1}$ (M3) sem carga (GROTTA, 2003), porém, com carga, diminuiu a velocidade, apresentando diferença estatística para as marchas estudadas, sendo maior em M3, o dobro em relação a M1 e M2. Para os preparos, a maior queda de velocidade foi no reduzido, cerca de $20 \%$. A exigência de força de tração na barra não sofreu influência dos tratamentos, valores semelhantes aos de SIQUEIRA et al. (2001), menores que SILVA (2000) e MARQUES (2002) e superiores a ASAE (1996) e MARQUES (1999).

A potência na barra apresentou interação significativa entre os fatores, como mostrado na Tabela 2. Observa-se que, nas marchas M1 e M2, o preparo do solo não teve influência, porém, na marcha M3, o preparo convencional exigiu maior potência em relação ao solo escarificado. Isso pode ser explicado pela maior patinagem e, conseqüentemente, menor velocidade nesse sistema; o plantio direto ficou em posição intermediária. Analisando cada preparo individualmente, observa-se que, no convencional, a menor exigência é na marcha M1 (menor velocidade), o mesmo ocorrendo para o plantio direto, porém, nesse, a marcha M2 foi igual a M1. Na escarificação, a exigência de potência não foi significativa para as marchas, o que se explica pelo fato de que, nesse preparo, ocorreu diminuição de $6 \%$ na velocidade de deslocamento, sendo os valores encontrados neste experimento superiores aos de FURLANI (2000). 
A capacidade de campo efetiva apresentou comportamento igual à variável velocidade, pois a largura útil de trabalho da semeadora foi igual em todas as parcelas. Os valores de capacidade de campo efetiva foram maiores que os encontrados por LEVIEN et al. (1999) e MARQUES et al. (1999).

TABELA 1. Síntese da análise de variância e do teste de médias para as variáveis velocidade de deslocamento, aproveitamento da velocidade nominal, força de tração, potência e capacidade de campo efetiva.

\begin{tabular}{|c|c|c|c|c|c|}
\hline Fatores & $\begin{array}{c}\text { Velocidade } \\
\left(\mathrm{km} \mathrm{h}^{-1}\right)\end{array}$ & $\begin{array}{c}\mathrm{Av}_{\text {nom }} \\
(\%)\end{array}$ & $\begin{array}{c}\text { Força de Tração } \\
(\mathrm{kN})\end{array}$ & $\begin{array}{c}\text { Potência } \\
(\mathrm{kW})\end{array}$ & $\begin{array}{c}\text { Ccef } \\
\left(\mathrm{ha} \mathrm{h}^{-1}\right)\end{array}$ \\
\hline \multicolumn{6}{|l|}{$\operatorname{Marcha}(\mathrm{M})$} \\
\hline M1 & $6,3 \mathrm{c}$ & $13,7 \mathrm{~b}$ & $13,6 \mathrm{a}$ & 23,8 & $2,3 \mathrm{c}$ \\
\hline M2 & $6,9 \mathrm{~b}$ & $14,8 \mathrm{~b}$ & $13,8 \mathrm{a}$ & 26,4 & $2,5 \mathrm{~b}$ \\
\hline M3 & $8,4 \mathrm{a}$ & $20,0 \mathrm{a}$ & $12,9 \mathrm{a}$ & 30,1 & $3,0 \mathrm{a}$ \\
\hline \multicolumn{6}{|l|}{ Preparo $(\mathrm{P})$} \\
\hline Convencional & $7,4 \mathrm{a}$ & $14,9 \mathrm{~b}$ & $13,0 \mathrm{a}$ & 26,7 & $2,7 \mathrm{a}$ \\
\hline Plantio direto & $7,4 \mathrm{a}$ & $14,9 \mathrm{~b}$ & $13,6 \mathrm{a}$ & 27,9 & $2,7 \mathrm{a}$ \\
\hline Reduzido & $7,0 \mathrm{~b}$ & $19,5 \mathrm{a}$ & $13,7 \mathrm{a}$ & 26,6 & $2,5 \mathrm{~b}$ \\
\hline \multicolumn{6}{|l|}{ Teste F } \\
\hline $\mathrm{M}$ & $257,7 *$ & $80,69 *$ & $0,92 \mathrm{~ns}$ & $12,94 *$ & $223,44 *$ \\
\hline $\mathrm{P}$ & $12,0 *$ & $12,02 *$ & $0,61 \mathrm{~ns}$ & $0,80 \mathrm{~ns}$ & $10,52 *$ \\
\hline$M \times P$ & $1,5 \mathrm{~ns}$ & $1,46 \mathrm{~ns}$ & $1,95 \mathrm{~ns}$ & $2,77 *$ & $2,30 \mathrm{~ns}$ \\
\hline C.V. (\%) & 3,2 & 16,4 & 12,1 & 11,7 & 3,4 \\
\hline
\end{tabular}

Médias seguidas de mesmas letras, minúsculas nas colunas, indicam que os dados não diferiram entre si, a $5 \%$ de probabilidade.

TABELA 2. Interação entre os fatores marcha e preparo para a variável potência na barra (kW).

\begin{tabular}{llll}
\hline Preparo & \multicolumn{3}{c}{ Marchas } \\
\cline { 2 - 4 } & \multicolumn{1}{c}{ M1 } & \multicolumn{1}{c}{ M2 } & M3 \\
\hline Convencional & 21,0 a B & 27,0 a A & 32,4 a A \\
Plantio direto & 25,1 a B & 26,4 a AB & 31,8 ab A \\
Reduzido & 25,3 a A & 26,4 a A & $26,7 \mathrm{~b} \mathrm{~A}$ \\
\hline
\end{tabular}

Médias seguidas de mesmas letras, minúsculas nas colunas e maiúsculas nas linhas, indicam que os dados não diferiram entre si, a $5 \%$ de probabilidade.

A rotação do motor (Tabela 3) foi menor na marcha M3, porém destaca-se que essa rotação é ideal de trabalho para esse trator, portanto, do ponto de vista do desempenho, essa seria a marcha indicada para essa operação.

O consumo horário de combustível aumentou da marcha M1 para M3; já os consumos por área e específico não foram influenciados pelas marchas, porém, nos diferentes preparos, todos os consumos estudados foram maiores em semeadura no solo escarificado, no qual se observa a menor velocidade e a maior patinagem. O consumo por área é semelhante ao de LEVIEN et al. (1999) e menor que o encontrado por MARQUES et al. (1999) e FURLANI (2000).

Os sistemas de preparo do solo não influenciaram na patinagem média dos rodados do trator para a marcha M1. No entanto, observou-se aumento significativo da patinagem no sistema reduzido para as marchas M2 e M3, resultados semelhantes aos encontrados por FURLANI (2000). 
TABELA 3. Síntese da análise de variância e do teste de médias para as variáveis rotação do motor e patinagem das rodas do trator.

\begin{tabular}{|c|c|c|c|c|c|}
\hline Fatores & \multirow{2}{*}{$\begin{array}{c}\text { Rotação do } \\
\text { Motor } \\
(\mathrm{rpm})\end{array}$} & \multicolumn{3}{|c|}{ Consumo de Combustível } & \multirow[b]{2}{*}{$\begin{array}{c}\text { Patinagem } \\
(\%)\end{array}$} \\
\hline Marcha (M) & & $\begin{array}{l}\text { Por área } \\
\left(\mathrm{L} \mathrm{ha}^{-1}\right)\end{array}$ & $\begin{array}{l}\text { Horário } \\
\left(\mathrm{L} \mathrm{h}^{-1}\right)\end{array}$ & $\begin{array}{l}\text { Específico } \\
\left(\mathrm{g} \mathrm{kWh}^{-1}\right)\end{array}$ & \\
\hline M1 & $2.089 \mathrm{a}$ & $5,7 \mathrm{a}$ & $12,9 \mathrm{c}$ & $459 \mathrm{a}$ & 4,7 \\
\hline M2 & $2.090 \mathrm{a}$ & $6,1 \mathrm{a}$ & $15,2 \mathrm{~b}$ & $482 \mathrm{a}$ & 7,2 \\
\hline M3 & $2.009 \mathrm{~b}$ & $5,8 \mathrm{a}$ & $17,4 \mathrm{a}$ & $483 \mathrm{a}$ & 6,8 \\
\hline \multicolumn{6}{|l|}{ Preparo $(\mathrm{P})$} \\
\hline Convencional & $2.087 \mathrm{a}$ & $5,5 \mathrm{~b}$ & $14,5 \mathrm{~b}$ & $457 \mathrm{~b}$ & 4,3 \\
\hline Plantio direto & $2.078 \mathrm{a}$ & $5,5 \mathrm{~b}$ & $14,7 \mathrm{~b}$ & $441 \mathrm{~b}$ & 4,9 \\
\hline Reduzido & $2.023 \mathrm{~b}$ & $6,5 \mathrm{a}$ & $16,3 \mathrm{a}$ & $526 \mathrm{a}$ & 9,4 \\
\hline \multicolumn{6}{|l|}{ Teste F } \\
\hline $\mathrm{M}$ & $10,10 *$ & $3,47 *$ & $67,07 *$ & $0,66 \mathrm{~ns}$ & $6,09 *$ \\
\hline $\mathrm{P}$ & $5,78 *$ & $20,16^{*}$ & $13,22 *$ & $7,64 *$ & $25,39 *$ \\
\hline $\mathrm{M} \times \mathrm{P}$ & $1,41 \mathrm{~ns}$ & $1,33 \mathrm{~ns}$ & $0,92 \mathrm{~ns}$ & $2,44 \mathrm{~ns}$ & $3,83^{*}$ \\
\hline C.V $(\%)$ & 2,4 & 7,9 & 6,3 & 11,9 & 30,6 \\
\hline
\end{tabular}

Médias seguidas de mesmas letras, minúsculas nas colunas, indicam que os dados não diferiram entre si, a $5 \%$ de probabilidade.

No geral, a operação de semeadura apresentou maiores dificuldades no preparo reduzido, pois o solo fica mais solto que no plantio direto e preparo convencional, onde as gradagens niveladoras deixam o mesmo mais consolidado.

TABELA 4. Interação entre os fatores marcha e preparo para a variável patinagem (\%).

\begin{tabular}{cccc}
\hline Preparo & \multicolumn{3}{c}{ Marchas } \\
\cline { 2 - 4 } & M1 & M2 & M3 \\
\hline Convencional & $3,3 \mathrm{a} \mathrm{A}$ & $5,6 \mathrm{~b} \mathrm{~A}$ & $4,1 \mathrm{~b} \mathrm{~A}$ \\
Plantio direto & $4,8 \mathrm{a} \mathrm{A}$ & $6,0 \mathrm{~b} \mathrm{~A}$ & $4,0 \mathrm{~b} \mathrm{~A}$ \\
Reduzido & $6,0 \mathrm{a} \mathrm{B}$ & $10,1 \mathrm{a} \mathrm{A}$ & $12,2 \mathrm{a} \mathrm{A}$ \\
\hline
\end{tabular}

Médias seguidas de mesmas letras, minúsculas nas colunas e maiúsculas nas linhas, indicam que os dados não diferiram entre si, a $5 \%$ de probabilidade.

\section{CONCLUSÕES}

No preparo reduzido do solo, a operação de semeadura apresentou maior consumo de combustível, menor velocidade de deslocamento, menor capacidade de campo efetiva e maior patinagem.

A semeadora-adubadora apresentou o pior desempenho no solo sob preparo reduzido, usandose a terceira marcha.

Em preparo convencional e plantio direto, a semeadora-adubadora apresentou bom desempenho, independentemente da marcha utilizada.

\section{REFERÊNCIAS}

AMERICAN SOCIETY OF AGRICULTURAL ENGINEERS. Terminology for soil-engaging components for conservation tillage planters, drilss and seedrs. In: . ASAE Standards 1996:

Standards Engineering Practices Data, St. Joseph, 1996. p.309-14. 
AMERICAN SOCIETY OF AGRICULTURAL ENGINEERS. Terminology and definitions for agricultural tillage implements. In: ASAE Standards 1997: Standards Engineering Practices Data, St. Joseph, 1997. p.254-75.

EMPRESA BRASILEIRA DE PESQUISA AGROPECUÁRIA. Sistema brasileiro de classificação de solos. Rio de Janeiro, 1999. 412 p.

FURLANI. C.E.A. Efeito do preparo do solo e do manejo da cobertura de inverno na produtividade da cultura do feijoeiro (Phaseolus vulgaris L.). 2000. 218 f. Tese (Doutorado em Energia na Agricultura) - Faculdade de Ciências Agronômicas, Universidade Estadual Paulista, Botucatu, 2000.

GADANHA JÚNIOR, C.D.; MOLIN, J.P.; COELHO, J.L.D.; YAHN, C.H.; TOMIMORI, S.M.A. Máquinas e implementos agrícolas do Brasil. São Paulo: Instituto de Pesquisas Tecnológicas do Estado de São Paulo, 1991. 468 p.

GROTTA, D.C.C. Desempenho de um trator agrícola em operação de gradagem utilizando biodiesel etílico filtrado de óleo residual como combustível. 2003. 44 f. Dissertação (Mestrado em Ciência do Solo) - Faculdade de Ciências Agrárias e Veterinárias, Universidade Estadual Paulista, Jaboticabal, 2003.

LEVIEN, R.; MARQUES, J.P.; BENEZ, S.H. Desempenho de uma semeadora-adubadora de precisão, em semeadura de milho (Zea mays L.), sob diferentes formas de manejo do solo. In: CONGRESSO BRASILEIRO DE ENGENHARIA AGRÍCOLA, 28., 1999, Pelotas. Anais... Pelotas: Sociedade Brasileira de Engenharia Agrícola, 1999. 1 CD-ROM.

LOPES, A.; FURLANI, C.E.A.; MELLO, J.G.S. Protótipo de sistema instrumental para medição de consumo de combustível em tratores. In: CONGRESSO BRASILEIRO DE INFORMÁTICA APLICADA À AGROPECUÁRIA E À INDUSTRIA, 4., 2003. Porto Seguro: Sociedade Brasileira de Informática Aplicada à Agropecuária e à Industria, 2003. 1 CD-ROM.

MARQUES, J.P. Manejo da vegetação espontânea para implantação da cultura do milho (Zea mays L.) em plantio direto e preparo convencional do solo. 1999. 98 f. Dissertação (Mestrado em Energia na Agricultura) - Faculdade de Ciências Agronômicas, Universidade Estadual Paulista, Botucatu, 1999.

MARQUES, J.P.; PONTES, J.R.V.; BENEZ, S.H. Desempenho de uma semeadora-adubadora de precisão na semeadura de milho (Zea mays L.) em preparo convencional do solo e em semeadura direta. In: CONGRESSO BRASILEIRO DE ENGENHARIA AGRÍCOLA, 28., 1999, Pelotas. Anais... Pelotas: Sociedade Brasileira de Engenharia Agrícola, 1999. 1 CD-ROM.

MARQUES, J.P. Efeito dos sistemas de manejo do solo e da cobertura de entressafra na cultura da soja (Glycine max L.). 2002. 224 f. Tese (Doutorado em Energia na Agricultura) - Faculdade de Ciência Agronômicas, Universidade Estadual Paulista, Botucatu, 2002.

SILVA, S.L. Avaliação de semeadoras para plantio direto: demanda energética, distribuição longitudinal e profundidade de deposição de sementes em diferentes velocidades de deslocamento. 2000. 123 f. Tese (Doutorado em Energia na Agricultura) - Faculdade de Ciências Agronômicas, Universidade Estadual Paulista, Botucatu, 2000.

SIQUEIRA, R.; ARAÚJO, A.G.; CASÃO JÚNIOR, R.; RALISH, R. Desempenho energético de semeadoras-adubadoras de plantio direto na implantação da cultura da soja (Glycine max L.). In: CONGRESSO BRASILEIRO DE ENGENHARIA AGRÍCOLA, 30., 2001, Foz do Iguaçu. Anais... Foz do Iguaçu: Sociedade Brasileira de Engenharia Agrícola, 2001. 1 CD-ROM. 